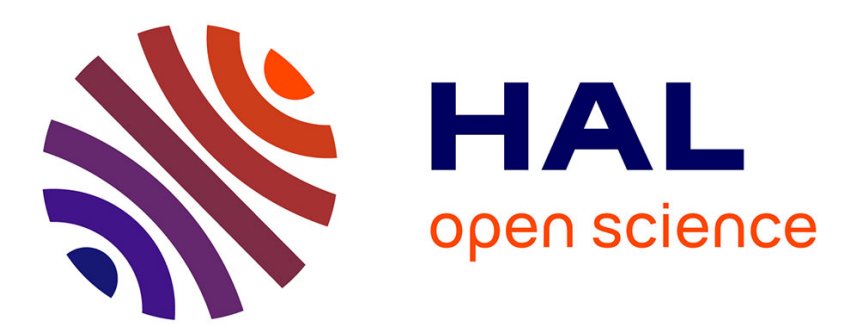

\title{
Radioactive ion beam physics at the cyclotron research centre Louvain-la-Neuve
}

\author{
Mark Huyse, Riccardo Raabe
}

\section{To cite this version:}

Mark Huyse, Riccardo Raabe. Radioactive ion beam physics at the cyclotron research centre Louvainla-Neuve. Journal of Physics G: Nuclear and Particle Physics, 2011, 38 (2), pp.24001. 10.1088/09543899/38/2/024001 . hal-00600871

\section{HAL Id: hal-00600871 https://hal.science/hal-00600871}

Submitted on 16 Jun 2011

HAL is a multi-disciplinary open access archive for the deposit and dissemination of scientific research documents, whether they are published or not. The documents may come from teaching and research institutions in France or abroad, or from public or private research centers.
L'archive ouverte pluridisciplinaire HAL, est destinée au dépôt et à la diffusion de documents scientifiques de niveau recherche, publiés ou non, émanant des établissements d'enseignement et de recherche français ou étrangers, des laboratoires publics ou privés. 


\title{
Radioactive Ion Beam Physics at the Cyclotron Research Centre Louvain-la-Neuve
}

\author{
Mark Huyse, Riccardo Raabe \\ Instituut voor Kern- en Stralingsfysica, Katholieke Universiteit Leuven, \\ BE-3001 Leuven, Belgium \\ E-mail: mark.huyse@fys.kuleuven.be, raabe@kuleuven.be
}

\begin{abstract}
.
The first beam of post-accelerated radioactive ions was realized in 1989 at the Louvainla-Neuve research facility. The method employed two coupled cyclotrons to produce, separate and re-accelerate the species of interest. Several technological challenges were solved in the process, to obtain pure and intense beams for use in nuclear physics research. Similarly, new techniques were developed and refined for the measurement of the nuclear reactions induced by the radioactive beams. The available energy range made the facility particularly suited for nuclear astrophysics studies, and important results were obtained in the determination of stellar reaction rates using beams of ${ }^{7} \mathrm{Be}$, ${ }^{13} \mathrm{~N},{ }^{18} \mathrm{~F},{ }^{18,19} \mathrm{Ne}$. A beam of ${ }^{6} \mathrm{He}$ ions was extensively used in studies of the nuclear structure (halos, molecular states) and dynamics (the reaction process at energies around the potential barrier).
\end{abstract}

\section{Introduction}

Started in 1987 through a collaboration between the Belgian universities of Louvain-laNeuve (UCL), Brussels (ULB) and Leuven (K.U.Leuven), the so-called RIB (Radioactive Ion Beam) project aimed to couple the two cyclotrons at Louvain-la-Neuve in order to produce an intense, energetic radioactive ion beam. On June 22, 1989, after two years of developments, a first beam was post-accelerated: a million ${ }^{13} \mathrm{~N}$ ions per second were accelerated to an energy of $8 \mathrm{MeV}$ [1]. In December 1990, a first experiment was carried out: in 33 hours of beam time the cross section of the proton-capture reaction on ${ }^{13} \mathrm{~N}$ was measured using a $3 \times 10^{8}$ particles per s (pps), pure $(\sim 99 \%) 8.2 \mathrm{MeV}^{13} \mathrm{~N}$ beam [2]. Since then various beams of radioactive isotopes ranging from ${ }^{6} \mathrm{He}\left(T_{1 / 2}=0.8 \mathrm{~s}\right)$ to ${ }^{35} \mathrm{Ar}$ $\left(T_{1 / 2}=1.7 \mathrm{~s}\right)$, with intensities from $1 \times 10^{5} \mathrm{pps}\left({ }^{35} \mathrm{Ar}\right)$ up to $2 \times 10^{9} \mathrm{pps}\left({ }^{19} \mathrm{Ne}\right)$, were developed and many experiments in nuclear astrophysics and nuclear structure were performed. Later on, the CYCLONE 44 cyclotron was designed and built specifically for experiments in nuclear astrophysics [3]. The RIB project came to an end in 2009.

Reaction studies with post-accelerated radioactive ion beams first of all require intense and pure beams at the right energy. The processes involved in arriving at this 


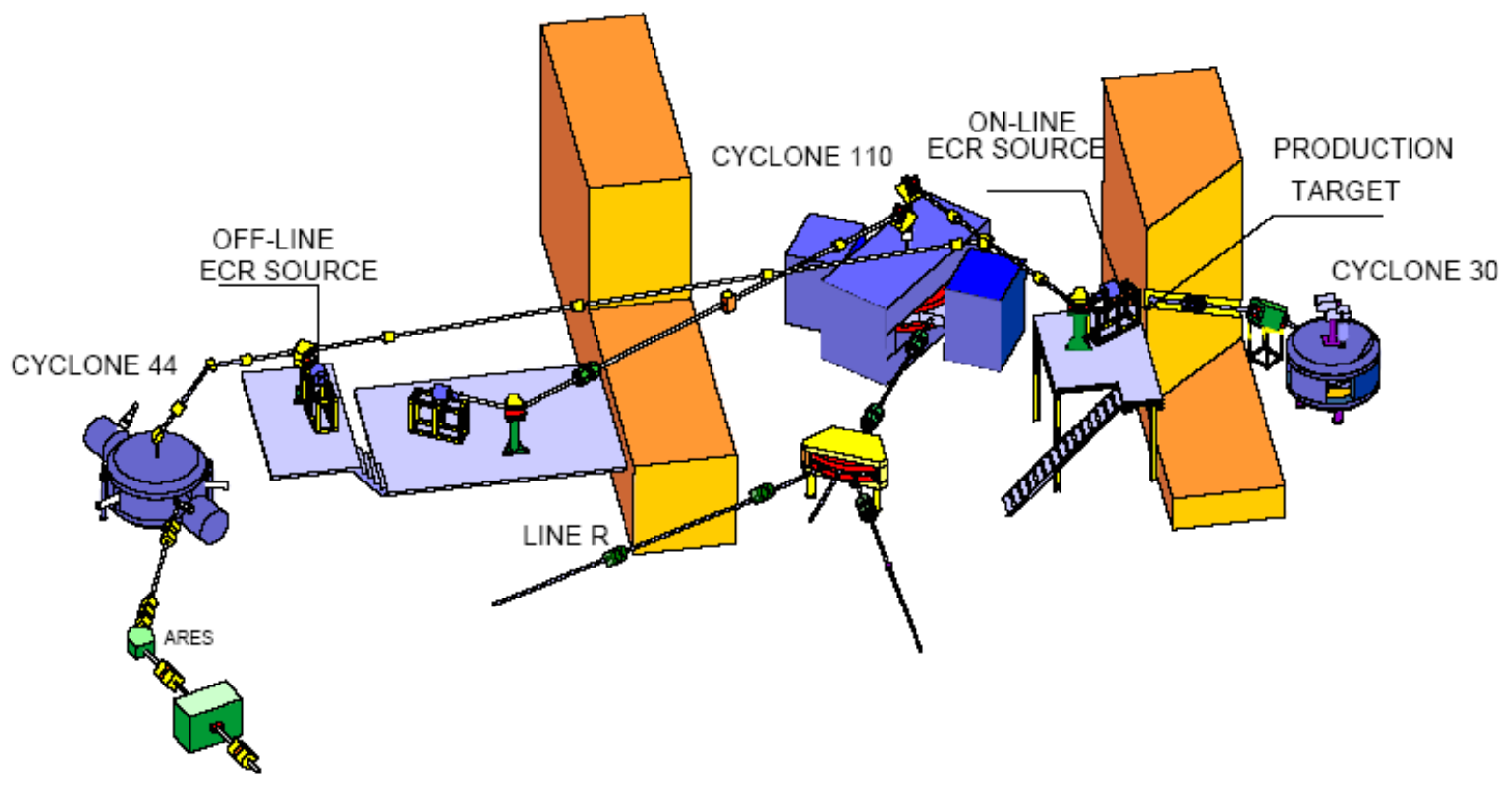

Figure 1. Layout of the radioactive ion beam facility in Louvain-la-Neuve. Both the CYCLONE 110 and CYCLONE 44 cyclotrons post-accelerated radioactive nuclei produced by reactions induced by the proton beam of CYCLONE 30.

goal are the primary production in the target, the release out of the target, the transport to the ion source, the ionization to the desired charge state, the post acceleration and final beam manipulation. At the secondary target an efficient and selective detection set up which is able to cope with the intrinsic radioactivity of the secondary beam is needed. As the RIB facility at Louvain-la-Neuve was the first to deal with these issues, a description of the different developments undertaken are given in section 2 . The following sections deal with nuclear astrophysics, nuclear structure and with reaction mechanisms around the Coulomb barrier studied at Louvan-la-Neuve using RIBs.

\section{The production of RIBs at Louvain-la-Neuve}

The layout of the RIB facility in Louvain-la-Neuve, which displays all the features of subsequent reaccelerated beam ISOL (Isotope-Separation-On-Line) facilities, is shown in Fig. 1. The coupled-cyclotron method is used to produce and post-accelerate the radioactive ion beams: the first cyclotron, CYCLONE 30, which is a $30 \mathrm{MeV}$ proton accelerator, developed for medical purposes [4], produces the desired unstable isotope in suitable targets. Beam intensities up to $300 \mu \mathrm{A}$ are used to irradiate the production target.

The resulting activity diffuses out of the hot target and is fed into an ECR ion source for ionization. The source operates at $6.4 \mathrm{GHz}$ and is designed to produce with a high efficiency ions in relatively low-charge states [5]. After a first magnetic separation in a low mass-resolving dipole, the ions are injected in the second cyclotron, CYCLONE 
110, for subsequent acceleration up to the desired energy. The publication, presenting the first beam obtained [1], describes all the ingredients of this process.

\subsection{The primary production}

The possibilities of producing radioactive species with the protons from CYCLONE 30 are limited to nuclei close to stability owing to the limited energy but one can produce very large quantities as the primary beam intensity can reach $300 \mu \mathrm{A}$. The choice of the production target is governed by the yield of the reaction, by the behavior of the target material under intense proton bombardment and by its release properties. The first beam developed at Louvain-la-Neuve was $10-\mathrm{min}^{13} \mathrm{~N}$ and its development will serve as a guideline to describe the whole RIB production process. A dedicated yield study considering two possible reactions for the production of ${ }^{13} \mathrm{~N}$ was set up [6]. The chosen reaction $\left(30-\mathrm{MeV} p+{ }^{13} \mathrm{C}\right)$ has a yield of $1.6{ }^{13} \mathrm{~N}$ nuclei per 1000 incoming protons.

\subsection{Target-ion source system and development of some beams}

Fast and efficient target-ion-source systems have been developed over the years at different ISOL facilities. However a special challenge was encountered at Louvain-laNeuve as the power delivered in the thick target by the $300 \mu \mathrm{A} 30 \mathrm{MeV}$ proton beam reaches $6 \mathrm{~kW}$, a record at that time. A robust target made of ${ }^{13} \mathrm{C}$ pellets, obtained by graphitizing $99 \%$ enriched ${ }^{13} \mathrm{C}$ powder was developed [7]. The release of the radioactive products out of the target was studied in off-line and on-line conditions [8]. The irradiated target not only releases the wanted radioactivity but also large quantities of unwanted radioactive and stable products. Chemical and/or cryogenic traps are used to reduce the contamination. The ion source needs to cope successfully with these hostile conditions in order to maintain its fast and efficient ionization. An electron cyclotron resonance (ECR) ion source was specifically developed for this and the efficiency and lingering time of different gasses studied [9]. In order to reduce the sticking, a quartz tube was installed in the source since quartz forms a rather inert surface. This resulted in an on-line efficiency for ${ }^{13} \mathrm{~N}$ of $8 \%$ [9]. Another strong point of ECR sources is the relative high-charge state that can be reached with good efficiency. This facilitates the post-acceleration process considerably. Furthermore their ion-optical characteristics are well adapted for injection into cyclotrons. Next to the fully on-line approach where the production with the CYCLONE 30, the extraction out of the target, the ionization, mass separation and post acceleration with CYCLONE 110 all follows in a fast and continuous mode leading to beams of radioisotopes as short-living as $800 \mathrm{~ms}$, a semi on-line mode was also developed for the production of $109.8-\mathrm{min}{ }^{18} \mathrm{~F}$ [10] and an off-line batch method for the production of 53-days ${ }^{7} \mathrm{Be}[11]$. The beams that were developed and post-accelerated at Louvain-la-Neuve are ${ }^{6} \mathrm{He},{ }^{7} \mathrm{Be},{ }^{10,11} \mathrm{C},{ }^{13} \mathrm{~N},{ }^{15} \mathrm{O},{ }^{18} \mathrm{~F},{ }^{18,19} \mathrm{Ne}$ and ${ }^{35} \mathrm{Ar}$. 


\subsection{Purity of post-accelerated RIBs}

Post-accelerated radioactive ion beams are mainly used for reaction studies. This imposes specific problems not encountered so dramatically with stable beams. RIBs are typically weak in intensity, sometimes contaminated and, of course, radioactive. The intensity depends on the reaction used and on the target-ion source system, both discussed above, and on the efficiency of the post acceleration. CYCLONE 110 reaches $10 \%$ efficiency for the acceleration of stable nitrogen while in on-line conditions the efficiency for ${ }^{13} \mathrm{~N}$ is $4 \%$ [1]. The ${ }^{13} \mathrm{~N}$ beam is $10^{3}-10^{4}$ times weaker than the isobaric contaminants ${ }^{13} \mathrm{C}$ and ${ }^{12} \mathrm{CH}$. The relative mass differences with the ${ }^{13} \mathrm{~N}$ ions are about $1.7 \times 10^{4}$. The ${ }^{12} \mathrm{CH}^{1+}$ molecular ions can be removed by employing a thin carbon foil where the molecular ions are dissociated and the products separated magnetically. The cyclotron can now be tuned as a radiofrequency mass spectrometer by reducing the acceleration voltage in order to increase the number of turns in the machine and therefore separates the ${ }^{13} \mathrm{C}$ contaminant from the ${ }^{13} \mathrm{~N}$ beam. This method results in an isobaric suppression factor of $10^{-6}$ while maintaining the efficient transmission through the cyclotron [12].

As the ${ }^{13} \mathrm{~N}$ beam was the first post-accelerated ISOL beam developed it is instructive to examine Table I of the article describing the production process [1]. There a comparison is made between the off-line efficiencies of all the production steps and the final on-line efficiencies. Compromises have to be made when the different steps are brought together and a stable system running over several days is the final goal. The expected production by combining all off-line determined parameters was $6 \times 10^{10}$ pps while on-line $1.5 \times 10^{8}$ pps was routine. Such a reduction is normal and should be taken in account when planning new facilities or experiments. Finally these results could only be achieved through a close collaboration between the accelerator engineers and the physicists.

\section{Nuclear astrophysics}

Understanding the role of nuclear processes in astrophysical sites is a topic of great current interest and addresses questions such as how energy is generated in stars and how the chemical elements are synthesized. The advent of radioactive ion beams has created new opportunities to measure directly important capture reaction rates or to obtain crucial information through indirect methods. A recent review on the impact of experiments with radioactive ion beams on the field of nuclear astrophysics can be found in Ref. [13]. In this section we will concentrate on the methods that were specifically developed for the reaction studies with RIBs, on the pioneering experiments at Louvainla-Neuve related to the hot hydrogen burning CNO cycle and its link to the NeNa mass region, and will conclude with a short overview of recent experiments. 


\subsection{Methods}

The reactions of astrophysical interest using RIBs in inverse kinematics impose specific requirements: firstly on the secondary target, and secondly on the detector set-up, which should be highly efficient as the secondary beam is in general of very low intensity and the reaction yield in the astrophysical energy domain is generally low. The detection set-up, however, must also cope with the radioactivity of the secondary beam. Finally reactions of astrophysical interest are typically measured in a well-defined energy domain.

As hydrogen and helium are the main ingredients of stars, reactions induced by these particles are of prime importance. However in order to study these reactions with RIBs, inverse kinematics has to be used implying the necessity of developing hydrogen and helium targets adapted to such experiments. For the ${ }^{13} \mathrm{~N}(p, \gamma)^{14} \mathrm{O}$ reaction a feasibility study using the ${ }^{13} \mathrm{C}(p, \gamma){ }^{14} \mathrm{~N}$ reaction was conducted comparing the resonance parameters obtained in inverse kinematics with an extended gas target (thintarget yield) and with a polyethylene foil (thick-target yield), with results from normal kinematics where thin and thick ${ }^{13} \mathrm{C}$ targets were bombarded with protons [14]. The loss in hydrogen of the polyethylene target was determined as a function of irradiation time and beam intensity ( $0.5 \%$ per hour per particle $\mathrm{nA})$.

Helium targets were developed by implantation in thin $\mathrm{Al}$ foils, where concentrations of $10^{17}-10^{18}$ at $/ \mathrm{cm}^{2}[15,16]$ were possible, and by using a gas-filled chamber with thin windows [17]. The latter was tested with the ${ }^{13} \mathrm{~N}(\alpha, p)^{16} \mathrm{O}$ reaction and compared to the inverse reaction ${ }^{16} \mathrm{O}(p, \alpha){ }^{13} \mathrm{~N}$.

There is no universal detection set-up for measuring reaction rates of astrophysical interest as all set-ups have to be tailored to the reaction under study. In the case of the ${ }^{13} \mathrm{~N}(p, \gamma){ }^{14} \mathrm{O}$ reaction a large volume Ge detector was preferred to $\mathrm{NaI}(\mathrm{Tl})$ detectors to record the $5.173 \mathrm{MeV}$ capture $\gamma$ rays $[9,12,14]$.

The amount of ${ }^{20} \mathrm{Na}$ produced in the ${ }^{19} \mathrm{Ne}(p, \gamma){ }^{20} \mathrm{Na}$ reaction was measured through the $\beta^{+}$-delayed $\alpha$ radioactivity of ${ }^{20} \mathrm{Na}$ using two different methods and through the $\beta^{+}$rays of ${ }^{20} \mathrm{Na}$. The key problem was to detect the rare ${ }^{20} \mathrm{Na}$ radioactivity in the very high background arising from the decay of the unreacted ${ }^{19} \mathrm{Ne}$ beam. The first set-up for the $\beta^{+}$-delayed $\alpha$ registration consisted of the on-line $\alpha$ detection in doublesided silicon strip detectors while the second set-up employed the off-line counting of nuclear tracks in polycarbonate solid state track detectors $[18,19]$. The detection of the $\beta^{+}$decay of ${ }^{20} \mathrm{Na}$ was performed with the STAR set-up (Solenoid and Telescope for Astrophysical Research) where the combination of a solenoidal field and a stack of plastic scintillators were used to discriminate the $\beta^{+}$signal from ${ }^{20} \mathrm{Na}$ in the background of the ${ }^{19} \mathrm{Ne}$ beam [20]. All set-ups were first tested with the ${ }^{19} \mathrm{Ne}(\mathrm{d}, \mathrm{n}){ }^{20} \mathrm{Na}$ reaction which has a considerably higher yield.

Another method which was also based on the detection of the radioactive end product of the reaction of interest (here the ${ }^{13} \mathrm{~N}(d, n)^{14} \mathrm{O}$ reaction) consisted of detecting with well-shielded large-volume Ge detectors, the $\gamma$ rays emitted in the $\beta^{+}$decay of ${ }^{14} \mathrm{O}$, implanted on a movable tape [21]. 
In a collaborative effort between the universities of Edinburgh and Louvain-laNeuve, the very successful highly-segmented LEDA charged-particle array was developed to detect, in a strong $\beta^{+}$background, relatively low-intensity charged reaction residues [22]. This approach has now been implemented at many places and has become one of the key experimental tools at such RIB facilities.

In a number of cases the sensitivity and selectivity of the detection set-ups described above were not sufficient and the Astrophysics REcoil Separator (ARES) was developed to measure $(p, \gamma)$ reactions [23]. ARES has been coupled to CYCLONE44, the cyclotron purpose built for RIBs in the astrophysics domain [3].

In principle, in order to measure the proton resonance parameters when using a thin secondary target, the energy of the radioactive beam should be varied. This is a time-consuming process and a method was developed to scan in one measurement many resonances by using thick polyethylene targets [24, 25]. The radioactive beam loses energy traversing the target: the initial beam energy and target thickness are chosen in order to cover the centre-of-mass energies of interest. The method, widely applied in Louvain-la-Neuve and other RIB facilities [2, 25, 26, 27, 28], has been extended to study the ground and excited states of unbound nuclei, either directly or through their isobaric analogue states: for example ${ }^{11} \mathrm{~N}$ [29] and ${ }^{19} \mathrm{Na}[30,31]$ in Louvain-la-Neuve, ${ }^{7} \mathrm{He}[32]$ at the TwinSol Facility of the Notre Dame University. In addition, using $\alpha$ particles as a target, the same method was applied to the investigation of the cluster structure of resonances in selected nuclei (section 4.2).

\subsection{First measurements}

At the start of the RIB project, the primary focus was to measure cross-sections of astrophysical importance with special emphasis on capture reaction sequences from the hot $\mathrm{CNO}$ cycle towards the $r p$ process. The reactions studied in Louvain-laNeuve are shown in Fig. 2. The first result and also the first capture measurement with a radioactive ion beam was the direct determination of the ${ }^{13} \mathrm{~N}(p, \gamma){ }^{14} \mathrm{O}$ reaction cross section which is crucial to determine the stellar conditions under which the hot CNO cycle competes with the cold CNO cycle $[2,12]$. In the relevant range of stellar temperatures the cross-section is dominated by the $\ell_{\mathrm{p}}=0$ resonance at $E_{\text {c.m. }}=0.545$ $\mathrm{MeV}$ corresponding to the $5.173 \mathrm{MeV}\left(1^{-}\right)$state in ${ }^{14} \mathrm{O}$. The $\Gamma_{\gamma}$ width obtained resulted in a rate twice that previously adopted. In order to fully address the consequences one must also determine the interference of this resonance with the non-resonant capture. Therefore, the proton spectroscopic factors for the non-resonant contribution were measured with the ${ }^{13} \mathrm{~N}(d, n)^{14} \mathrm{O}$ reaction [21]. No other experiments on the proton capture of ${ }^{13} \mathrm{~N}$ were performed with post-accelerated ISOL beams elsewhere. This is probably due to the fact that the intensities reached at Louvain-la-Neuve (up to 15 particles pA) were already very high. This crucial reaction has been the object of further studies using the in-flight technique at Texas A\&M [33], RIKEN [34] and Beijing [35], in particular on the non-resonant part. The dominant uncertainty in the ${ }^{13} \mathrm{~N}(p, \gamma){ }^{14} \mathrm{O}$ 


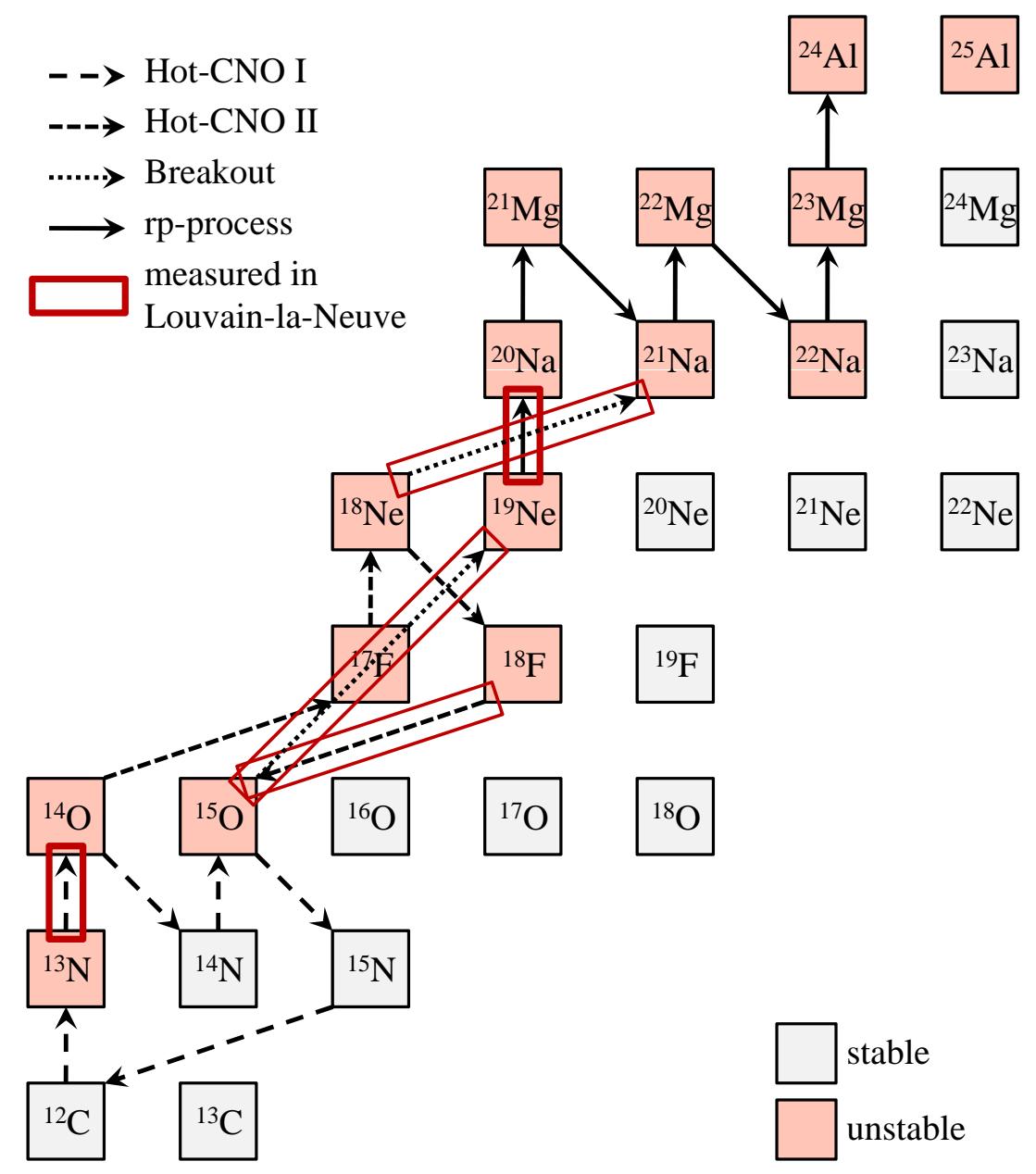

Figure 2. Portion of the chart of nuclei, with indication of the paths of the hot-CNO cycle (Hot-CNO I) and very-hot CNO cycle (Hot-CNO II), with the possible escape routes. The reactions studied in Louvain-la-Neuve are highlighted.

reaction rate presently arises from the $\gamma$ width of the $5.173 \mathrm{MeV}$ state in ${ }^{14} \mathrm{O}$.

Shortly after these first experiments with the ${ }^{13} \mathrm{~N}$ beam, a second important reaction was tackled. In a collaboration with the University of Edinburgh the influence of the ${ }^{19} \mathrm{Ne}(p, \gamma){ }^{20} \mathrm{Na}$ reaction on breakout of the CNO cycles into the $r p$ process was studied. The reaction rate is believed to be dominated by a resonance at $E_{\text {c.m. }}=0.447 \mathrm{MeV}$ corresponding to the $2.643 \mathrm{MeV}$ state in ${ }^{20} \mathrm{Na}$, the first level above threshold in the ${ }^{19} \mathrm{Ne}(p, \gamma){ }^{20} \mathrm{Na}$ reaction. The $2.643 \mathrm{MeV}$ state in ${ }^{20} \mathrm{Na}$ was previously observed in nuclear reactions induced by stable beams, such as ${ }^{20} \mathrm{Ne}\left({ }^{3} \mathrm{He}, t\right){ }^{20} \mathrm{Na}$ and ${ }^{20} \mathrm{Ne}(p, n){ }^{20} \mathrm{Na}$, but the different results are in disagreement which leads to uncertainty in the spin assignment. Depending on the spin assignment the resonance strength estimates vary by almost a factor of 10 , but all are considerably lower than the strength of the ${ }^{13} \mathrm{~N}(p, \gamma){ }^{14} \mathrm{O}$ reaction. The resonant scattering in a thick target method described in section 3.1 was first employed to investigate resonances between 650 and $1000 \mathrm{keV}$ [25]. Following this, a series of experiments, all based on the detection of the decay of ${ }^{20} \mathrm{Na}$, were performed 
in the 1990's where more and more stringent limits were set on the resonant strength of the low-lying resonances in ${ }^{20} \mathrm{Na}[18,36,37]$. These measurements suffered from a low detection efficiency $(\leq 1.5 \%)$ and the presence of a background from the ${ }^{19} \mathrm{Ne}(d, n){ }^{20} \mathrm{Na}$ reaction induced by the beam on the deuterium present in the $\left(\mathrm{CH}_{2}\right)_{\mathrm{n}}$ target. The ARES spectrometer was used to overcome these problems and an upper limit of $\omega \gamma \leq 15.2 \mathrm{meV}$ for the resonance strength of the state at $447 \mathrm{keV}$ was obtained [38]. The astrophysical implications of these studies show that the breakout of the CNO cycle to the $r p$ process will be determined by the preceding ${ }^{15} \mathrm{O}(\alpha, \gamma){ }^{19} \mathrm{Ne}$ reaction.

\subsection{Further measurements}

Following on from the pioneering work with ${ }^{13} \mathrm{Ne}$, many more beams have been developed of which ${ }^{7} \mathrm{Be},{ }^{18} \mathrm{~F},{ }^{18} \mathrm{Ne}$ have also been used for astrophysical studies. Furthermore, owing to the versatility of the LEDA array in its different configurations, not only could the direct measurement of $(p, \alpha)$ and $(\alpha, p)$ reactions be made, but resonance properties from elastic and inelastic scattering experiments could also be obtained (see also section 4).

The ${ }^{7}$ Be beam produced in batch mode has made it possible to measure the ${ }^{7} \mathrm{Be}+$ $p$ elastic cross section in the c.m. energy region from 0.3 to $0.75 \mathrm{MeV}$. From an $R$-matrix analysis of the cross section data, the energy and the width of the $1^{+}$resonance in ${ }^{8} \mathrm{~B}$ $\left(E_{x}=0.77 \mathrm{MeV}\right)$ was deduced and the astrophysical consequences discussed [39].

The ${ }^{18} \mathrm{~F}$ beam was used for a direct measurement of the ${ }^{18} \mathrm{~F}(p, \alpha){ }^{15} \mathrm{O}$ reaction. This reaction, which influences the destruction of ${ }^{18} \mathrm{~F}$, is important for nova $\gamma$-ray astronomy as during nova outbursts the decay of ${ }^{18} \mathrm{~F}$ is the main source of $\gamma$-ray emission at and below $511 \mathrm{keV}$. A series of experiments focussed on reactions with $E_{\text {c.m. }}$ above 550 $\mathrm{keV}[40,41,42]$. Evidence for a resonance at $330 \mathrm{keV}$ was found; the strength was later measured at the HRBIF facility [27]. The latest work at Louvain-la-Neuve yielded cross sections down to $E_{\text {c.m. }}=400 \mathrm{keV}$ constraining interference effects between $3 / 2^{+}$ resonances in ${ }^{19} \mathrm{Ne}$ [43]. However, recently a new experiment was performed with the ${ }^{19} \mathrm{Ne}$ beam to identify proton emitting states in ${ }^{19} \mathrm{Ne}$ through the inelastic scattering reaction ${ }^{19} \mathrm{Ne}(p, p){ }^{19} \mathrm{Ne}^{*}(p){ }^{18} \mathrm{~F}$. Several proton-emitting states were identified and a new broad $1 / 2^{+}$resonance was found, the low energy tail of which contributes to a significant enhancement of the ${ }^{18} \mathrm{~F}$ destruction rate at nova temperatures (see above). This reduces significantly the chance to observe $\gamma$-ray emission of ${ }^{18} \mathrm{~F}$ from a nearby nova explosion with existing telescopes [44].

The ${ }^{18} \mathrm{~F}$ beam was also used in the ${ }^{18} \mathrm{~F}(d, p){ }^{19} \mathrm{~F}^{*}$ reaction feeding levels above the $\alpha$ separation energy leading to ${ }^{15} \mathrm{~N}+\alpha$. The resulting spectroscopic factors were used to put new limits to the contribution of low-energy resonances to the rate of ${ }^{18} \mathrm{~F}(p, \alpha){ }^{15} \mathrm{O}$ [45].

The ${ }^{18} \mathrm{Ne}$ beam was used in a study of the ${ }^{18} \mathrm{Ne}(\alpha, p){ }^{21} \mathrm{Na}$ reaction, which can, at high stellar temperatures, be a possible breakout path out of the CNO cycle. Two centerof-mass energy domains were covered: between 2.04 and $3.01 \mathrm{MeV}$ [17] and between 1.7 
and $2.9 \mathrm{MeV}$ [46]. Upper and lower limits on the reaction rates have been calculated from the resonance parameters of newly identified states.

Also using the ${ }^{18} \mathrm{Ne}$ beam, an attempt was made at studying the states in ${ }^{19} \mathrm{Ne}$ relevant for the ${ }^{15} \mathrm{O}(\alpha, \gamma){ }^{19} \mathrm{Ne}$ reaction rate, by populating them through the $d\left({ }^{18} \mathrm{Ne},{ }^{19} \mathrm{Ne}\right) p$ reaction [47]. With the increase in beam intensities expected at the forthcoming facilities, this method will become competitive with stable beam studies.

\section{Nuclear structure}

The contribution of radioactive ion beams to nuclear structure research has been particularly significant in the region of light nuclei. Nuclei at and beyond the driplines have become accessible using reactions with exotic beams. The proximity of the driplines has led to the discovery of a number of features, appearing in nuclei at excitation energies close to and beyond the breakup threshold.

Research in this region at Louvain-la-Neuve has been supported by the availability, since 1994, of a beam of post-accelerated ${ }^{6} \mathrm{He}$, with an intensity and purity which were not matched elsewhere for many years. The beam has been directly used for investigations of the halo in ${ }^{6} \mathrm{He}$ and its role in the reaction process, but also as a tool to access exotic states and resonances in light systems.

\subsection{The halo of ${ }^{6} \mathrm{He}$}

The research on the two-neutron halo of ${ }^{6} \mathrm{He}$ in Louvain-la-Neuve focused especially on the effects induced on the reaction mechanism; such aspects are discussed further in section 5. Here we mention two experiments aiming at the clarification of the structure of the halo, in particular the correlations between the two halo neutrons.

Halos had been initially studied through breakup reactions at intermediate and high energies, looking at the momentum distribution of the fragments [48, 49]. The relatively low-energy post-accelerated beam at Louvain-la-Neuve opened the possibility for the use of transfer reactions. Because of the characteristics of the two neutrons in the halo - a low binding energy and a narrow structure of the wavefunction in momentum space transfer reactions with small momentum transfer and vanishing $Q$-value are favoured. The maximum cross section is thus obtained at low incident energies, for transfer into excited states of a stable target.

Given the $\alpha+2 n$ structure ${ }^{6} \mathrm{He}$, it was natural to look into two-neutron transfer reactions, as they could be related to the spatial configuration of the two halo neutrons: the so-called di-neutron and cigar configurations [50]. Transfer reactions were performed on ${ }^{12} \mathrm{C}[51],{ }^{1} \mathrm{H}[52]$ and ${ }^{4} \mathrm{He}$ targets $[53,54]$. All experiments employed the LEDA array; the former two focused on the study of the reaction mechanism, while the latter also investigated the importance of the transfer in relation to the ${ }^{6} \mathrm{He}$ configuration. The use of ${ }^{4} \mathrm{He}$ as a target had several consequences. Because of the symmetry of the system, the elastic scattering and the transfer of two neutrons would contribute to the same exit 


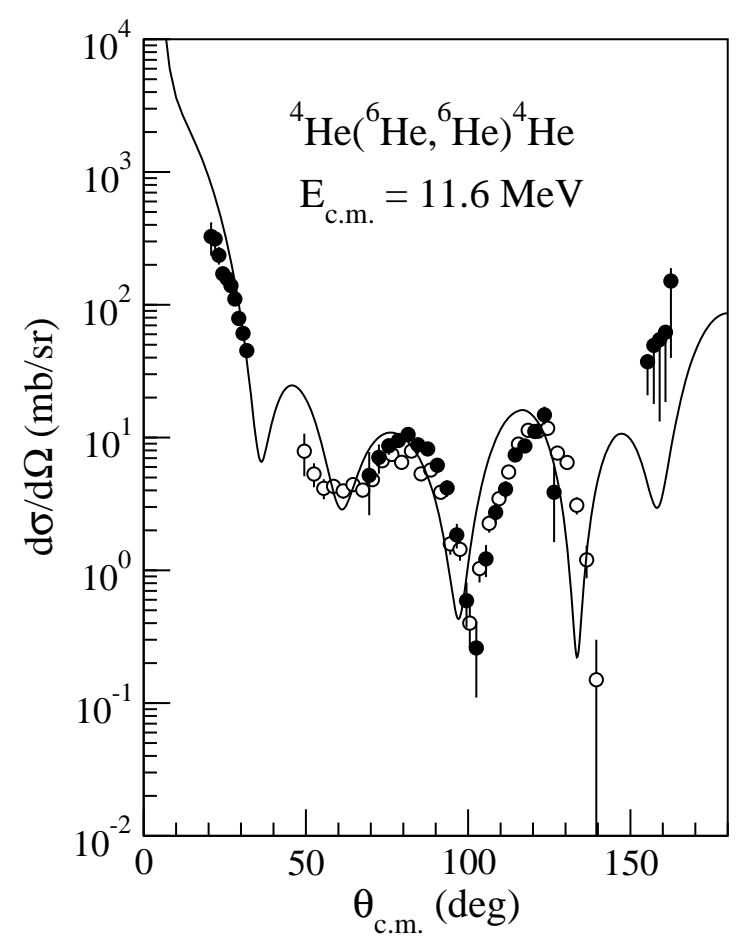

Figure 3. ${ }^{4} \mathrm{He}\left({ }^{6} \mathrm{He},{ }^{6} \mathrm{He}\right){ }^{4} \mathrm{He}$ cross section at $E_{\text {c.m. }}=11.6 \mathrm{MeV}$, measured using a gas target (open dots) [53] and a ${ }^{4} \mathrm{He}$-implanted $\mathrm{Al}$ target (full dots) [54]. The curve is a coherent sum of elastic scattering and transfer amplitudes, the latter obtained with a DWBA calculation.

channel (a ${ }^{6} \mathrm{He}$ nucleus and an $\alpha$ particle), with the transfer manifesting itself through interference phenomena and especially a rise of the elastic scattering cross section at backward centre-of-mass angles. A gas cell was first employed, but this limited the angular range at which the outgoing particles could be detected. This improved with the used of a ${ }^{4} \mathrm{He}$-implanted $\mathrm{Al}$ foil [15], a novelty at the time in combination with a radioactive ion beam. The second measurement indeed revealed ${ }^{6} \mathrm{He}+\alpha$ events at large centre-of-mass angles (see Fig. 3) proving the occurrence of two-neutron transfer. In the original work [54], data were reasonably well reproduced by a distorted-wave Born approximation (DWBA) calculation, assuming a direct transfer of two neutrons as a cluster. Later works used these data and those obtained at a higher energy at the Flerov Institute in Dubna [55], to investigate in more detail the possible sequential transfer of the two neutrons, which could have originated from a cigar configuration of the halo neutrons. In both Refs. [56] and [57] it was concluded that the direct transfer is the dominant mechanism, allowing the weight of the di-neutron component in the halo to be estimated to be around $95 \%$ [57].

Another process which was identified as strongly dependent on the details of the halo is the $\beta$-decay of ${ }^{6} \mathrm{He}$, in particular the deuteron-emission channel, first observed at ISOLDE [58]. The reason of the very weak branching ratio $\left(\sim 10^{-6}\right)$ was explained in Ref. [59] in terms of a cancellation between two parts of the Gamow-Teller matrix 
element, related to the internal and external (halo) regions of the ${ }^{6} \mathrm{He}$ ground state and the $\alpha+\mathrm{d}$ scattering wave functions. Measurements of the branching ratio performed at ISOLDE [58, 60] and TRIUMF [61] using low-energy beams suffered from normalization uncertainties. In Louvain-la-Neuve, the pure post-accelerated beam permitted the technique of direct implantation in a silicon detector [62], where decays could be counted with $100 \%$ efficiency providing an accurate normalization, to be employed. A problem that had to be overcome was the instantaneous suppression of the beam to a level much better than the branching ratio, $10^{-6}$, required in order to observe the decay events without beam-induced background. This was achieved by a combination of collimators and a rotating shutter wheel. The branching ratio $(1.65 \pm 0.10) \times 10^{6}$ [63] was measured with an uncertainty that represented a significant improvement with respect to previous results. This, and the related deuteron energy spectrum obtained, can provide accurate tests of models of the ${ }^{6} \mathrm{He}$ ground-state wave function $[63,64]$. The techniques developed here were later employed at TRIUMF to study the $\beta$-delayed deuteron emission of ${ }^{11} \mathrm{Li}$ [65].

\subsection{Cluster states}

The possibility of states in light nuclei, with configurations reminiscent of molecular structures, was advanced as early as 1938 [66]. In such cases, nucleons exchanged between different $\alpha$ clusters ensure the binding of the system. The existence of these structures was shown in ${ }^{9} \mathrm{Be}$ through the identification of rotational bands built on the deformed molecular $\alpha: n: \alpha$ state $[67,68]$. An analogue identification of states in ${ }^{10} \mathrm{Be}$ has been more elusive. Of particular interest would be a $\alpha: 2 n: \alpha$ state in which the two neutrons occupy a $\sigma^{2}$ valence orbital: owing to Pauli blocking, the neutrons would push the $\alpha$ clusters apart resulting in a very deformed state. A $0^{+}$state at $E^{*}=6.18 \mathrm{MeV}$ was the main candidate, with the $2^{+}$excited state of the corresponding rotational band at $E^{*}=7.54 \mathrm{MeV}$. But conclusive evidence could only come from the identification and characterization of the $4^{+}$state.

This was obtained in two separate measurements at Louvain-la-Neuve. A first indication came from the study of the ${ }^{6} \mathrm{He}+{ }^{6} \mathrm{Li}$ reaction at $18 \mathrm{MeV}$ beam energy [69]: a state at $10.2 \mathrm{MeV}$ in ${ }^{10} \mathrm{Be}$, populated by the transfer of an $\alpha$ particle from the target and identified via the coincident detection of the outgoing ${ }^{6} \mathrm{He}, \alpha$ and $d$ particles, was assigned as $4^{+}$by analysing the angular correlation between the products. A following experiment [70] concentrated on the characteristics of this resonance, which was studied using the method of resonant elastic scattering in a thick gas target. A $7.5-\mathrm{MeV}^{6} \mathrm{He}^{1+}$ beam impinged on a volume of ${ }^{4} \mathrm{He}$ gas; the beam particles lose energy traversing the gas volume, undergoing elastic scattering with enhanced probability for center-of-mass energies corresponding to the resonance (see Fig. 4). The coincidence detection of the ${ }^{6} \mathrm{He}$ and ${ }^{4} \mathrm{He}$ particles and their angular correlation allowed to definitely assign the $4^{+}$ spin to the state, identifying it as a member of the rotational band. The cross section indicated a very large $\alpha$-particle component of the state (one of the largest $\alpha$-cluster 

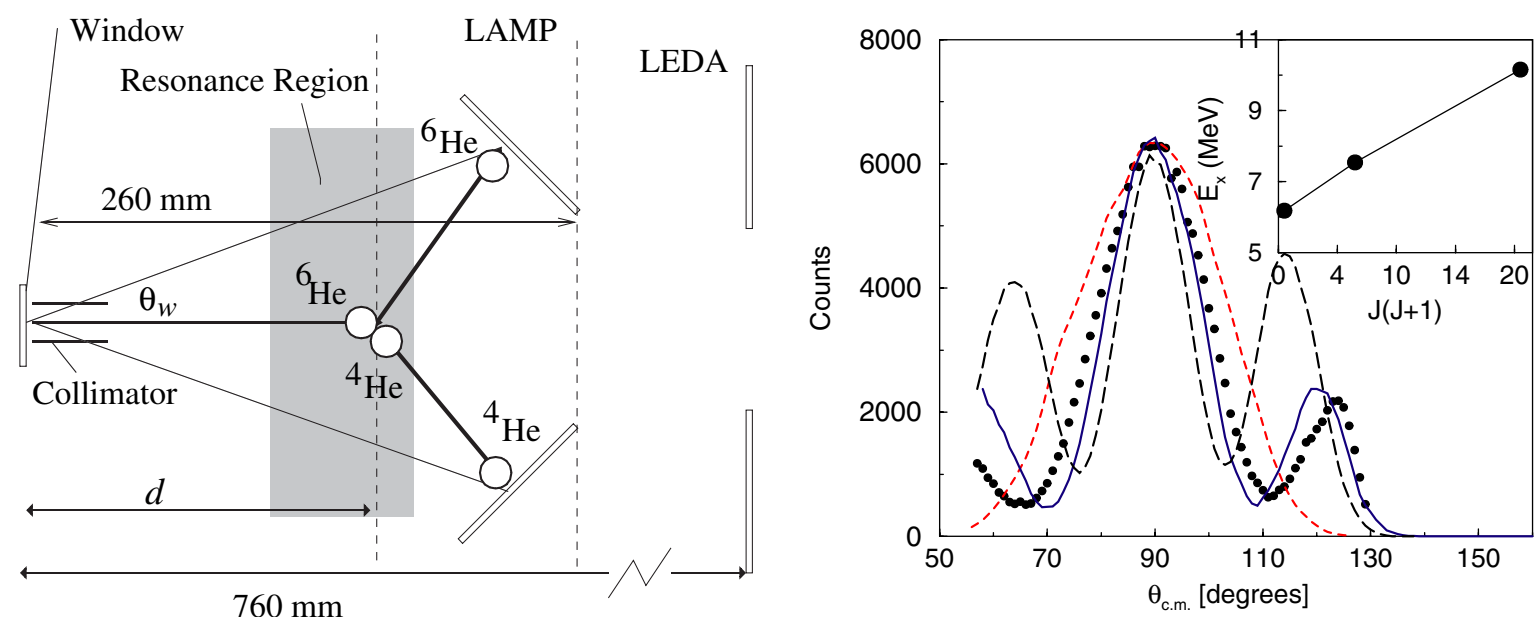

Figure 4. (from Ref. [70]). Left: schematic view of the experimental set-up used to study the $10.15-\mathrm{MeV}$ state in ${ }^{10} \mathrm{Be}$ : the ${ }^{6} \mathrm{He}$ beam loses energy traversing the ${ }^{4} \mathrm{He}$ gas, undergoing elastic scattering in the region corresponding to the resonance. Right: the center-of-mass distribution of the detected particles is compared to simulations corresponding to different spin values of the resonance, showing the $4^{+}$character of the state. The inset show the energy-spin systematics of the band based on the $0^{+}$ state at $6.18 \mathrm{MeV}$.

spectroscopic factors known to date). Finally, from its energy, measured at $10.15 \mathrm{MeV}$, a large moment of inertia was derived, confirming the very deformed $\alpha: 2 n: \alpha$ nature of the state at $6.18 \mathrm{MeV}$.

The same experimental technique was used with the ${ }^{18} \mathrm{Ne}$ beam to investigate $\alpha$ cluster states in ${ }^{22} \mathrm{Mg}[71]$. A comparison with mirror states in ${ }^{22} \mathrm{Ne}$ showed significant, poorly understood differences.

In other measurements, these structures in light nuclei were investigated by identifying "quasi-free" scattering events on clusters of the target. This was for example the case for the scattering of ${ }^{6} \mathrm{He}$ off deuterons in ${ }^{6} \mathrm{Li}[72]$ and off $\alpha$ particles in ${ }^{9} \mathrm{Be}[73]$.

\subsection{In-beam spectroscopy using RIBs}

Thanks to the early availability of very intense post-accelerated radioactive ion beams, Louvain-la-Neuve has been a favourite place to test novel techniques in nuclear spectroscopy. An interesting example is provided by the $\gamma$-ray spectroscopy of nuclei produced in fusion-evaporation reactions. The high-spin states in such nuclei are usually reached using intense stable beams and different regions of the nuclear chart have been studied that way. However, some regions are not easily accessible using stable targetbeam combinations. As an example, the study region of the heavy, unstable $N \sim Z$ nuclei is hindered by the very low cross sections for multiple neutron evaporation. The use of a proton-rich radioactive ion beam could be an advantage as the corresponding cross sections are higher, provided that this increase compensates the lower beam intensity. An additional problem of such an approach is represented by the high $511-\mathrm{keV}$ 
background in the $\gamma$ detectors, originating from the $\beta^{+}$decay of the radioactive beam.

A measurement was performed using a very intense $\left(10^{9} \mathrm{pps}\right) 70-\mathrm{MeV}{ }^{19} \mathrm{Ne}^{4+}$ beam impinging on a ${ }^{40} \mathrm{Ca}$ target $[74,75]$, with the purpose of investigating these issues and test possibilities for reducing the background. The $\gamma$ rays produced in fusion-evaporation reactions were detected in an array of escape-suppressed Ge detectors surrounding the target. Additionally, the LEDA array was employed to detect evaporated light charged particles.

Several conclusions were drawn: it was necessary to carefully shield the Comptonsuppression detectors from the radiation produced by the decay of beam particles hitting collimators close to the target; from this, the importance of a very-well focused beam without a halo was identified. The charged-particle array proved very effective in identifying the reaction channels; here, particle discrimination (protons and $\alpha$ 's) was crucial, determining an ideal thickness of the detector of about $150 \mu \mathrm{m}$. Finally, the most effective background suppression was obtained by the time correlation between the $\gamma$ rays and the beam particles. To achieve this, the beam was pulsed in 2 ns-wide bursts separated by 73 ns. The $\gamma$-ray spectra measured in between pulses were scaled and subtracted from the coincidence spectra, eliminating most of the random background. The effect is clearly visible in Fig. 5. At present, this technique is widely used at RIB facilities, including, for example, REX-ISOLDE to reduce the background in the Miniball $\gamma$-ray array in Coulex and transfer measurements.

\section{Reaction mechanism around the barrier}

As already recognized with the measurements that led to the discovery of the halo $[76,77]$, light exotic nuclei have properties that significantly influence the reaction mechanism. The characteristics of these nuclei could enhance the cross section for selected direct channels (for example, breakup or neutron transfer). At energies around the potential barrier, where the terms connecting the different exit channels of the scattering are no longer negligible with respect to the kinetic energy of the particles, coupling effects become important modifying the probability for elastic scattering [78] and fusion [79, 80]. The latter process in particular was the centre of much interest shortly after the discovery of halo nuclei, as it was speculated that the large spatial extension of neutron matter could lead to a large enhancement of the fusion probability.

The measurements related to these features are extremely challenging. For elastic scattering, a high accuracy is required, to allow to be compared with models which take into account the various coupling effects. For fusion, the small cross section around and below the potential barrier makes it inherently difficult to perform studies using the relatively low-intensity radioactive ion beams. For this reason, only limited regions of the excitation functions have been explored. Nevertheless, significant results have been achieved: recent reviews are given in Ref. [81] for elastic scattering and Refs. [82, 83] for fusion. The facility at Louvain-la-Neuve has contributed significantly to measurements using the ${ }^{6} \mathrm{He}$ (see below) and ${ }^{7}$ Be beams [84]. 


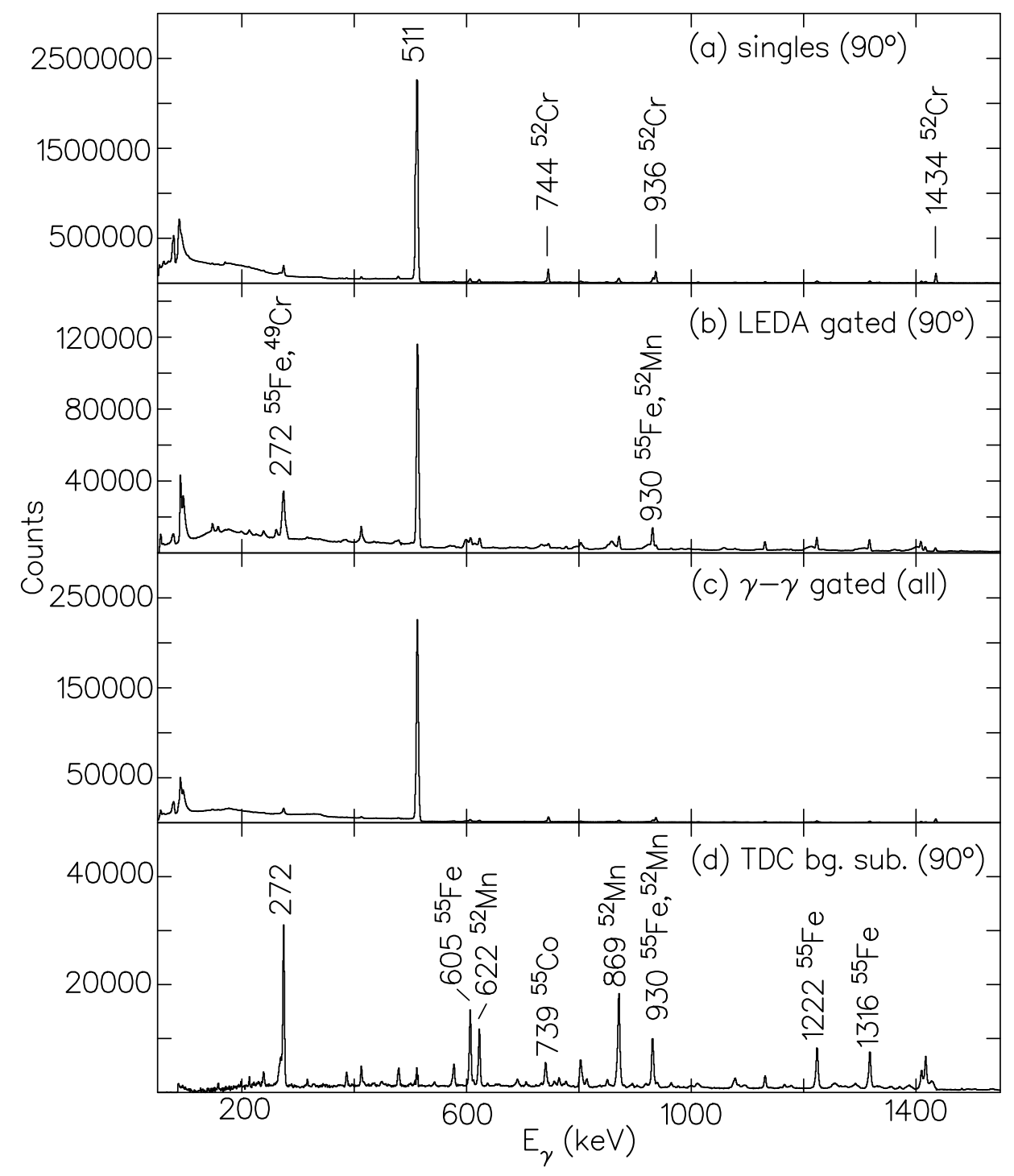

Figure 5. (from Ref. [74]). $\gamma$-ray energy spectra from the ${ }^{19} \mathrm{Ne}+{ }^{40} \mathrm{Ca}$ reaction. Panel (d) shows the dramatic improvement when the random background is subtracted using the time correlation with the beam pulses, as compared to singles (a), coincidence with light particles (b) and $\gamma-\gamma$ coincidences (c).

\subsection{Elastic scattering}

Elastic scattering of the ${ }^{6} \mathrm{He}$ beam on various thin targets were already obtained as a by-product of the first transfer measurements in the second half of the 1990's. These data have been the object of some re-analyses [51, 85, 86, 87, 88].

Those first measurements were carried out using the LEDA array, covering only forward angles up to about 75 degrees. Improved, dedicated measurements [89] employed additional detectors at backward angles to increase the angular coverage. The focus has been on the scattering of ${ }^{6} \mathrm{He}$ on ${ }^{208} \mathrm{~Pb}$ : the target was chosen because of its relatively simple and well-known structure, and because it would enhance Coulomb effects. The 


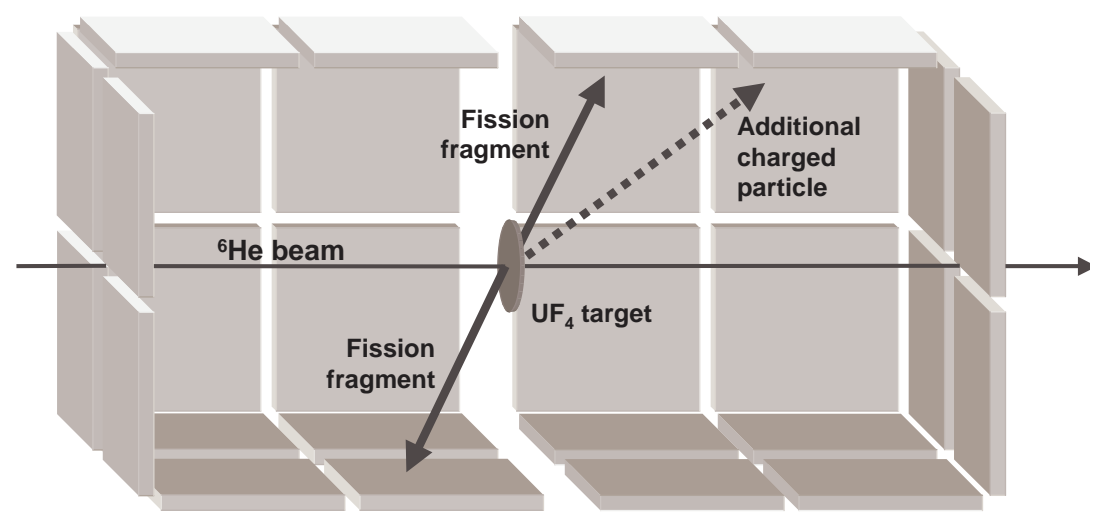

Figure 6. (from Ref. [92]). Schematic view of the set-up used in the measurement of the ${ }^{6} \mathrm{He}+{ }^{238} \mathrm{U}$ reaction. For clarity, some of the forty detectors are omitted in the drawing.

elastic cross section was measured at various centre-of-mass energies between 14 and 22 $\mathrm{MeV}$ and angles up to 170 degrees, and analyzed using optical model calculations. The data showed significant differences with respect to those obtained with ${ }^{6} \mathrm{Li}$ : in particular evidence for long range absorption was found, due to a polarization of the projectile in the Coulomb field of the target. A separate study of the outgoing $\alpha$ particles [90] showed the presence of a strong two-neutron transfer from the ${ }^{6} \mathrm{He}$ projectile to weakly bound states in ${ }^{210} \mathrm{~Pb}$.

The peculiarities in the elastic scattering of ${ }^{6} \mathrm{He}$ were also clearly highlighted in the comparison of ${ }^{6} \mathrm{He}+{ }^{64} \mathrm{Zn}$ and ${ }^{4} \mathrm{He}+{ }^{64} \mathrm{Zn}$ data, obtained in a campaign where the fusion cross section was also measured [91].

\subsection{Fusion measurements}

The measurement of the fusion cross section of the ${ }^{6} \mathrm{He}$ represents one of the highlights of the research at Louvain-la-Neuve using radioactive beams. Data were obtained on two targets, ${ }^{238} \mathrm{U}$ and ${ }^{64} \mathrm{Zn}$, utilizing different techniques. In both cases, the simultaneous measurement of large cross sections due to direct channels showed their importance at energies even below the barrier.

In each case, the nature of the target provided the means to identify the fusion process. The low fission barrier of ${ }^{238} \mathrm{U}$ meant that reactions in the thin $\mathrm{UF}_{4}$ target and the ${ }^{6} \mathrm{He}$ projectiles led to the back-to-back emission of two fission fragments. The fragments were identified by a coincident detection in a silicon array covering almost the full solid angle (Fig. 6). Fission could be induced by fusion, but also by other processes transferring sufficient energy to the target; in the latter cases, however, an additional light quasi-projectile particle would be emitted in coincidence and detected in the silicon array. This provided a way to separate the contribution of fusion from that of other direct processes. A first measurement with a reduced angular coverage showed a very large fission cross section [93]. The addition of detectors at backward angles proved, 


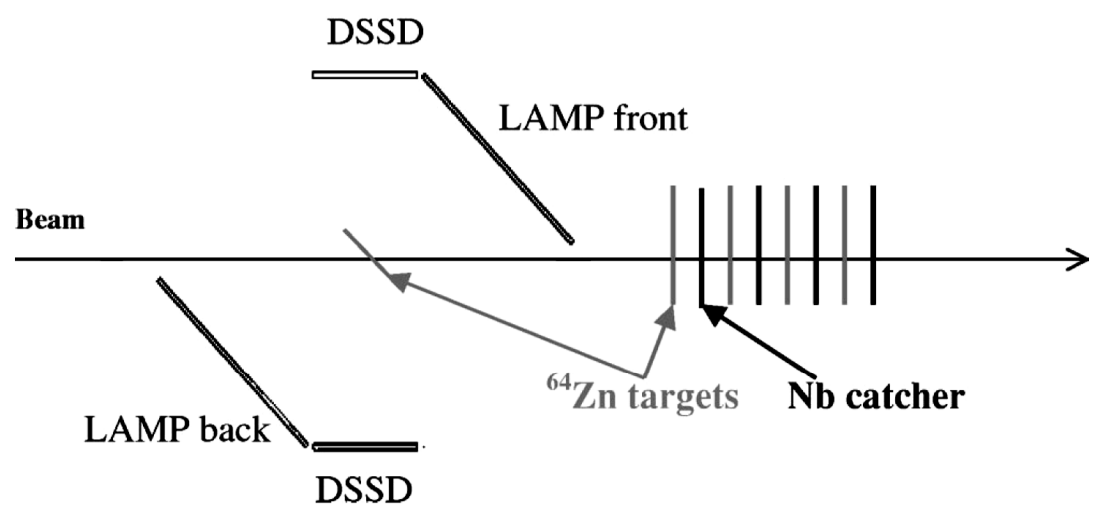

Figure 7. (from Ref. [94]). Schematic view of the set-up used in the measurement of ${ }^{6} \mathrm{He}+{ }^{64} \mathrm{Zn}$. LAMP and DSSD are arrays of silicon detectors.

however, the strong presence of direct processes, reducing the fusion cross section to levels comparable with those expected for a well-bound, non-halo projectile such as the stable ${ }^{4} \mathrm{He}[92]$.

With the ${ }^{64} \mathrm{Zn}$ target nucleus, an activation technique, followed by the off-line detection of residues, was employed. The ${ }^{64} \mathrm{Zn}$ provided the possibility of uniquely identifying the reaction products. In this experiment a stack of $\mathrm{Zn}$ targets was used, alternated with $\mathrm{Nb}$ foils that would catch the nuclei recoiling from the target (Fig. 7). Owing to the energy loss of the ${ }^{6} \mathrm{He}$ projectiles in traversing the stack, the reactions on each target would provide the cross section at a different energy, allowing the excitation function to be explored with only a few primary beam energies. An additional thin target, placed before the stack and surrounded by silicon detectors, served for the measurement of the elastic scattering as mentioned in the previous section [91]. A fusion reaction within the stack would eventually produce radioactive, longliving Ge, Ga and $\mathrm{Zn}$ evaporation residues. The atomic activity was identified and counted through the detection of the x-ray emission following the electron-capture decay; further identification of the specific isotope was obtained by its characteristics half-life signature. The results, reported in Ref. [94], confirmed that which was observed with the heavier ${ }^{238} \mathrm{U}$ target: the data for the fusion cross section did not show any appreciable enhancement with respect to those measured with ${ }^{4} \mathrm{He}$. On the other hand, a very large yield for ${ }^{65} \mathrm{Zn}$, not reproduced by fusion-evaporation calculations, could be explained by a large transfer and/or breakup cross section.

\section{Conclusions}

With the first post-acceleration of a radioactive ion beam, ${ }^{13} \mathrm{~N}$, research in nuclear physics acquired a powerful new tool to explore regions of the chart of nuclei far from stability. Coupling of the ISOL method for the on-line production and selection of ions with an efficient post-accelerator provided beams of unprecedented intensity and optical properties. To reach this seminal result, a number of technologies and solutions were 
conceived and pioneered at the Louvain-la-Neuve facility. These included methods to cope with the high power dissipated by the primary proton beam in the thick production target; research, on target materials and on suitable reactions, to obtain high production rates for unstable isotopes; and the means for optimizing the beam quality, in particular its purity, without compromising the overall acceleration efficiency. This experience has especially shown how entangled all these aspects are, and thus how the development of each beam requires a dedicated and specialized effort. This is an effort that has to be undertaken in close collaboration between the accelerator engineers and the physicists as the beam quality determines the detection set-up to be developed and vice-versa.

The development of new beams was driven by the physics program. Owing to the energies provided by the post-accelerator, nuclear reactions of astrophysical interest occurring at stellar temperatures were accessible. Important measurements were made of rates of reactions in the hot-CNO cycle and escapes towards the $r p$ process; clearly, such reactions - like ${ }^{13} \mathrm{~N}(p, \gamma){ }^{14} \mathrm{O},{ }^{19} \mathrm{Ne}(p, \gamma){ }^{20} \mathrm{Na}$ - were measured directly for the first time. A major role was then played by the ${ }^{6} \mathrm{He}$ beam, for research in nuclear structure and dynamics. On one hand, the focus has been on the characteristics of the two-neutron halo of ${ }^{6} \mathrm{He}$ and its influence on the reaction process at energies around the Coulomb barrier. On the other hand, the beam was used as a tool in reactions to produce systems in exotic states and resonances, such as the molecular structure of the very deformed $\alpha: 2 n: \alpha$ cluster state identified in ${ }^{10} \mathrm{Be}$.

As well as the beam production techniques, detection methods were developed and applied to counter the problems presented by the use of radioactive beams. The method of resonant scattering in a thick target in inverse kinematics was developed using solid targets (polyethylene, He-implanted foils) as well as He gas targets. The issues related to in-beam $\gamma$-ray spectroscopy with RIBs were carefully studied, producing reference results relevant for the subsequent design of optimized detection set-ups and experiments elsewhere.

Several of the methods pioneered in Louvain-la-Neuve have been taken up and further improved at the more recently-developed RIB facilities. With the progress of the latter, the activities in Louvain-la-Neuve have lessened; this is true in particular for nuclear structure research, which at present requires an increasingly strong concentration of resources. The RIB project came to an end in 2009. The experience accumulated in more than twenty years of target and ion source development continues, however, to be exploited by participation in projects such as beta-beams [95]; in particular for the aspects related to the production of extremely intense ${ }^{6} \mathrm{He}$ and ${ }^{18} \mathrm{Ne}$ beams - for which Louvain-la-Neuve has long held the records, and also for the more recent schemes involving ${ }^{8} \mathrm{Li}$ and ${ }^{8} \mathrm{~B}$ beams [96], where, once again, major technological challenges will need to be overcome. 


\section{Acknowledgments}

The authors wish to thank the staff of the Cyclotron Research Centre in Louvainla-Neuve for their continuous effort in developing and delivering excellent radioactive ion beams and for their warm hospitality in receiving the many outside users. We also wish to thank our colleagues from UCL, ULB and K.U.Leuven who formed the enthusiastic core of the facility. Finally, we acknowledge the continuous support from the Interuniversity Poles of Attraction Program from the Belgian State and from the various European programs supporting experiments at the facility.

\section{References}

[1] D. Darquennes et al., Phys. Rev. C 42, R804 (1990).

[2] P. Decrock et al., Phys. Rev. Lett. 67, 808 (1991).

[3] G. Ryckewaert et al., Nucl. Phys. A 701, 323c (2002).

[4] Y. Jongen et al., Nucl. Instrum. Methods Phys. Res. 24-25, 813 (1987).

[5] P. Decrock et al., Rev. Sci. Instrum. 61, 279 (1990).

[6] S. Wa-Kitwanga, P. Leleux, P. Lipnik, and J. Vanhorenbeeck, Phys. Rev. C 40, 35 (1989).

[7] D. Darquennes, T. Delbar, and P. Lipnik, Nucl. Instrum. Methods Phys. Res. B 47, 311 (1990).

[8] P. Decrock et al., Nucl. Instrum. Methods Phys. Res. B 70, 182 (1992).

[9] P. Decrock et al., Nucl. Instrum. Methods Phys. Res. B 58, 252 (1991).

[10] M. Cogneau et al., Nucl. Instrum. Methods Phys. Res. a 420, 489 (1999).

[11] M. Gaelens, M. Cogneau, M. Loiselet, and G. Ryckewaert, Nucl. Instrum. Methods Phys. Res. B 204, 48 (2003).

[12] T. Delbar et al., Phys. Rev. C 48, 3088 (1993).

[13] C. A. Bertulani and A. Gade, Physics Reports 485, 195 (2010).

[14] W. Galster et al., Phys. Rev. C 44, 2776 (1991).

[15] L. Weissman et al., Nucl. Instrum. Methods Phys. Res. B 170, 266 (2000).

[16] F. Vanderbist et al., Nucl. Instrum. Methods Phys. Res. B 197, 165 (2002).

[17] W. Bradfield-Smith et al., Phys. Rev. C 59, 3402 (1999).

[18] R. D. Page et al., Phys. Rev. Lett. 73, 3066 (1994).

[19] G. Vancraeynest et al., Nucl. Phys. A 616, 107c (1997).

[20] C. Michotte et al., Nucl. Instrum. Methods Phys. Res. A 366, 155 (1995).

[21] P. Decrock et al., Phys. Rev. C 48, 2057 (1993).

[22] T. Davinson et al., Nucl. Instrum. Methods Phys. Res. A 454, 350 (2000).

[23] M. Couder et al., Nucl. Instrum. Methods Phys. Res. A 506, 26 (2003).

[24] T. Delbar et al., Nucl. Phys. A 542, 263 (1992).

[25] R. Coszach et al., Phys. Rev. C 50, 1695 (1994).

[26] G. V. Rogachev et al., Phys. Rev. C 64, 061601 (2001).

[27] D. W. Bardayan et al., Phys. Rev. Lett. 89, 262501 (2002).

[28] J. J. He et al., Phys. Rev. C 80, 042801 (2009).

[29] E. Casarejos et al., Phys. Rev. C 73, 014319 (2006).

[30] C. Angulo et al., Phys. Rev. C 67, 014308 (2003).

[31] M. G. Pellegriti et al., Phys. Lett. B 659, 864 (2008).

[32] G. V. Rogachev et al., Phys. Rev. Lett. 92, 232502 (2004).

[33] X. Tang et al., Phys. Rev. C 69, 055807 (2004).

[34] T. Teranishi et al., Phys. Lett. B 650, 129 (2007).

[35] Z. H. Li et al., Phys. Rev. C 74, 035801 (2006).

[36] C. Michotte et al., Phys. Lett. B 381, 402 (1996). 
[37] G. Vancraeynest et al., Phys. Rev. C 57, 2711 (1998).

[38] M. Couder et al., Phys. Rev. C 69, 022801(R) (2004).

[39] C. Angulo et al., Nucl. Phys. A 716, 211 (2003).

[40] R. Coszach et al., Phys. Lett. B 353, 184 (1995).

[41] J. S. Graulich et al., Nucl. Phys. A 626, 751 (1997).

[42] J. S. Graulich et al., Phys. Rev. C 63, 011302 (2001).

[43] N. De Sereville et al., Phys. Rev. C 79, 015801 (2009).

[44] J. C. Dalouzy et al., Phys. Rev. Lett. 102, 162503 (2009).

[45] N. de Sereville et al., Phys. Rev. C 67, 052801(R) (2003).

[46] D. Groombridge et al., Phys. Rev. C 66, 055802 (2002).

[47] A. M. Laird et al., Phys. Rev. C 66, 048801 (2002).

[48] N. A. Orr et al., Phys. Rev. Lett. 69, 2050 (1992).

[49] A. A. Korsheninnikov and T. Kobayashi, Nucl. Phys. A 567, 97 (1994).

[50] M. V. Zhukov et al., Phys. Rep. 231, 151 (1993).

[51] A. N. Ostrowski et al., Phys. Rev. C 60, 064603 (1999).

[52] A. N. Ostrowski et al., Phys. Rev. C 63, 024605 (2001).

[53] R. Raabe et al., Phys. Lett. B 458, 1 (1999).

[54] R. Raabe et al., Phys. Rev. C 67, 044602 (2003).

[55] G. M. Ter-Akopian et al., Phys. Lett. B 426, 251 (1998).

[56] D. T. Khoa and W. von Oertzen, Phys. Lett. B 595, 193 (2004).

[57] L. I. Galanina and N. S. Zelenskaya, Phys. Atomic Nuclei 70, 283 (2007).

[58] K. Riisager et al., Phys. Lett. B 235, 30 (1990).

[59] D. Baye, Y. Suzuki, and P. Descouvemont, Prog. Theor. Phys. 91, 271 (1994).

[60] M. J. G. Borge et al., Nucl. Phys. A 560, 664 (1993).

[61] D. Anthony et al., Phys. Rev. C 65, 034310 (2002).

[62] D. Smirnov et al., Nucl. Instrum. Methods Phys. Res. A 547, 480 (2005).

[63] R. Raabe et al., Phys. Rev. C 80, 054307 (2009).

[64] E. M. Tursunov, D. Baye, and P. Descouvemont, Phys. Rev. C 73, 014303 (2006).

[65] R. Raabe et al., Phys. Rev. Lett. 101, 212501 (2008).

[66] L. R. Hafstad and E. Teller, Phys. Rev. 54, 681 (1938).

[67] W. von Oertzen, Z. Phys. A 354, 37 (1996).

[68] W. von Oertzen, Z. Phys. A 357, 355 (1997).

[69] M. Milin et al., Nucl. Phys. A 753, 263 (2005).

[70] M. Freer et al., Phys. Rev. Lett. 96, 042501 (2006).

[71] V. Z. Goldberg et al., Phys. Rev. C 69, 024602 (2004).

[72] D. Miljanic et al., Europhys. Lett. 76, 801 (2006).

[73] M. Majer et al., Eur. Phys. J. A 43, 153 (2010).

[74] W. N. Catford et al., Nucl. Instrum. Methods Phys. Res. A 371, 449 (1996).

[75] W. N. Catford et al., Nucl. Phys. A 616, 303c (1997).

[76] I. Tanihata et al., Phys. Rev. Lett. 55, 2676 (1985).

[77] I. Tanihata et al., Phys. Lett. B 160, 380 (1985).

[78] G. R. Satchler, Introduction to nuclear reactions, 2nd ed. (1990).

[79] M. Beckerman, Phys. Rep. 129, 145 (1985).

[80] S. G. Steadman and M. J. Rhoades-Brown, Ann. Rev. Nucl. Part. Sci. 36, 649 (1986).

[81] N. Keeley, N. Alamanos, K. W. Kemper, and K. Rusek, Prog. Part. Nucl. Phys. 63, 396 (2009).

[82] L. F. Canto, P. R. S. Gomes, R. Donangelo, and M. S. Hussein, Phys. Rep. 424, 1 (2006).

[83] N. Keeley, R. Raabe, N. Alamanos, and J. L. Sida, Prog. Part. Nucl. Phys., 59, 579 (2007).

[84] R. Raabe et al., Phys. Rev. C 74, 044606 (2006).

[85] O. R. Kakuee et al., Nucl. Phys. A 728, 339 (2003).

[86] K. Rusek, N. Keeley, K. W. Kemper, and R. Raabe, Phys. Rev. C 67, 041604(R) (2003).

[87] K. Rusek et al., Phys. Rev. C 72, 037603 (2005). 
[88] O. R. Kakuee et al., Nucl. Phys. A 765, 294 (2006).

[89] A. M. Sanchez-Benitez et al., Nucl. Phys. A 803, 30 (2008).

[90] D. Escrig et al., Nucl. Phys. A 792, 2 (2007).

[91] A. Di Pietro et al., Europhys. Lett. 64, 309 (2003).

[92] R. Raabe et al., Nature (London) 431, 823 (2004).

[93] M. Trotta et al., Phys. Rev. Lett. 84, 2342 (2000).

[94] A. Di Pietro et al., Phys. Rev. C 69, 044613 (2004).

[95] C. Volpe, J. Phys. G: Nucl. Part. Phys. 34, R1 (2007).

[96] C. Rubbia, A. Ferrari, Y. Kadi, and V. Vlachoudis, Nucl. Instrum. Methods Phys. Res. A 568, 475 (2006). 